\title{
COMPUTATIONAL FLUID DYNAMICS INVESTIGATION ON MULTIPLE INJECTOR CONCEPTS AT DIFFERENT SWIRL RATIOS IN A HEAVY DUTY ENGINE
}

\author{
Gustav Nyrenstedt', Moez Ben Houidi ${ }^{1}$, Rafig Babayev ${ }^{1}$, Hong Im ${ }^{1}$, Bengt Johansson ${ }^{1}$ \\ ${ }^{1}$ King Abdullah University of Science and Technology, Clean Combustion Research Center, Thuwal, \\ 23900, Saudi Arabia
}

\begin{abstract}
Numerical studies investigated how multiple injectors can reduce the high heat losses associated with swirl, as a further attempt to enhance thermal efficiencies of high-pressure combustion engines. Computational fluid dynamics simulations employed the Reynolds-averaged Navier-Stokes approach for one, two -and three injector configurations. High and medium load conditions were simulated at different swirl ratios. In general, an increased swirl ratio reduced engine efficiency. However, for all swirl ratios, three injectors provided higher efficiency. Two injectors decreased the heat losses for all swirl ratios, and injection against the swirl with multiple injectors provided high efficiencies. In combination with a waste heat recovery system, the two-injector case delivered an efficiency increase of $2.2 \%$-points for the medium load case. Three injectors delivered high efficiencies at all swirl ratios as an effect of a high flow rate and low heat losses. The multiple injector configurations evaluated in this study proved non-beneficial for the high load case. Spray-to-spray interactions lowered the combustion -and indicated efficiencies. However, the three injector case showed potential for delivering high indicated efficiency, from an increased flow rate, at high loads.
\end{abstract}

Keywords: Diesel Engine, Swirl, High Efficiency, Multiple Injectors

\author{
NOMENCLATURE \\ AMR Adaptive Mesh Refinement \\ AS2 Injection against a swirl ratio of 2 \\ ATDC After Top Dead Center \\ DCEE Double Compression Expansion Engine \\ EXMEP Exhaust Mean Effective Pressure \\ HCCI Homogenous Charge Compression Ignition \\ IMEP Indicated Mean Effective Pressure \\ LTC Low-Temperature Combustion \\ MEP Mean Effective Pressure \\ NS No Swirl \\ PPC Partially Premixed Combustion \\ RANS Reynolds-Averaged Navier-Stokes \\ RoHR Rate of Heat Release \\ S1 Swirl Ratio 1 \\ S2 Swirl Ratio 2 \\ S3 Swirl Ratio 3 \\ S5 Swirl Ratio 5 \\ SOI Start of Injection \\ WHR Waste Heat Recovery
}

\section{INTRODUCTION}

Global warming effects give increased priority to reduce greenhouse gas emissions [1]. Thus, the compression-ignition internal combustion engine, which contributes to greenhouse gas emissions, faces ever-more stringent emission regulations [2], demanding higher engine efficiency [3].

A fast heat release achieves high engine efficiency due to the resemblance of isochoric heat release [4]. Higher turbulence, from, for example, elevated in-cylinder swirl, renders a higher heat release rate for compression-ignition engines [5]. A study reported an efficiency increase of $2 \%$-points for a light-duty compression-ignition engine with an increased swirl ratio from 0 to 3 [6]. However, studies also suggest increased heat losses by swirl, leaving room for further improvement [7]. 
Multiple injector concepts reduce the heat losses for compression ignition engines by low near-wall temperature gradients, a smaller wall area covered by hot gases, and reduced near-wall velocity and turbulence [8-11]. Studies suggest a $25 \%$ heat loss reduction (at some load conditions without swirl) by placing two injectors at the rim of the piston bowl [12]. However, no study has been performed on how multiple injectors reduce heat losses at different swirl ratios.

This study aims to investigate how multiple injectors can mitigate heat losses and increase efficiency when combined with different swirl ratios. The baseline engine uses a double compression expansion engine (DCEE) approach [13]. The DCEE employs a split-cycle consisting of one low-pressure (LP) unit and one high-pressure (HP) unit (see FIGURE 1). The DCEE delivers efficiency up to $56 \%$ as a result of the high expansion ratio; high-pressure combustion; and the ability to recover exhaust energy, following from the split-cycle approach [14].

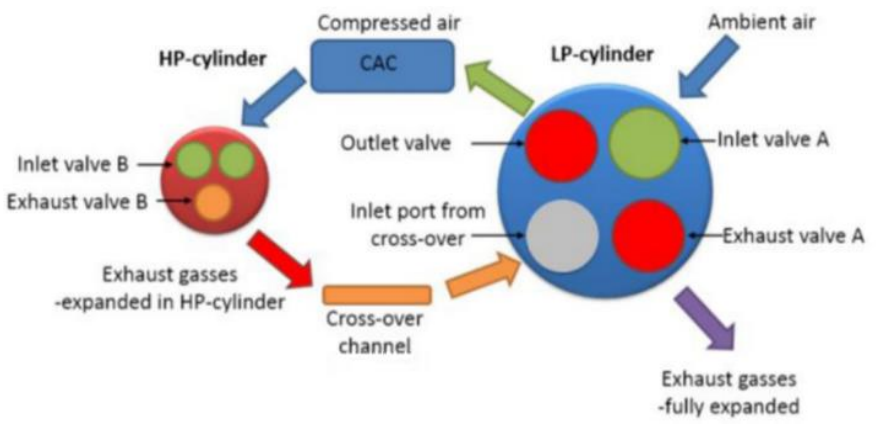

FIGURE 1: DCEE LAYOUT [14]

This study focuses on the DCEE HP-cylinder, a standard 4stroke engine with a low compression ratio. Thus, the study applies to conventional compression-ignition engines with the addition that the normally wasted exhaust energy can be utilized for generating useful work.

\section{METHODOLOGY}

Three-dimensional Reynolds-Averaged Navier-Stokes (RANS) simulations were performed using the Converge CFD software, version 2.4. The simulations included investigations of one, two, and three injectors at five different swirl ratios. This section covers some definitions; the computational setup and validation; and the test conditions.

\subsection{Performance Calculations}

This study uses several performance indicators described here. Energy levels normalized by the displacement volume define the mean effective pressures (MEP). We calculated: the FuelMEP from the lower heating value and the fuel mass; the combustion efficiency by dividing the combustion heat with the fuel energy; and the work output and gross indicated mean effective pressure $\left(\mathrm{IMEP}_{\mathrm{g}}\right.$ ) by integrating the pressure over the volume from inlet valve closing to exhaust valve opening. The difference between fuel energy, work output, and heat losses provides exhaust energy. Previous studies offer more details on the indicators presented here [11].

\subsection{CFD Setup}

The computational setup consisted of the combustion chamber surrounded by the cylinder head, liner, and piston boundaries. The head boundary was stationary while the piston boundary translated according to the piston motion characteristics of the test engine. The sub-models included a renormalization group (RNG) k-epsilon RANS model for turbulence closure. Adaptive mesh refinement (AMR), based on temperature and velocity gradients, was applied on the $2 \mathrm{~mm}$ base mesh. Zone-specific mesh embeddings were added to the injectors and walls to accurately resolve the near-nozzle and near-wall fluid flow. FIGURE 2 gives further insight into the meshing strategy at three different CADs for a case with swirl ratio 1 . The middle and bottom part of the figure reveals the AMR in combustion zones and fuel-rich zones.

The fuel injection consisted of spherical liquid diesel droplet parcels. Upon evaporation, the n-heptane emerged as a diesel surrogate to simplify chemical kinetics calculations. However, the models used the liquid properties and lower heating value of diesel. The spray breakup followed a hybrid Kevin-Helmholtz and Rayleigh-Taylor approach [15]. A Frossling evaporation model [16] estimated the evaporated droplet diameter.

The boundary temperature was assumed constant at $500 \mathrm{~K}$ for all simulations performed. This assumption is in line with wall-temperature measurements in engine operation, where the temperature changes are minor [17]. The Amsden model [18] estimated convective heat transfer losses while no thermal radiation model was utilized.

The chemistry calculations consisted of a 110 species nheptane mechanism [19] while a SAGE model [20] calculated the reaction source terms. All other computational sub-models follow the standards and recommendations from CONVERGE v2.4 [1].

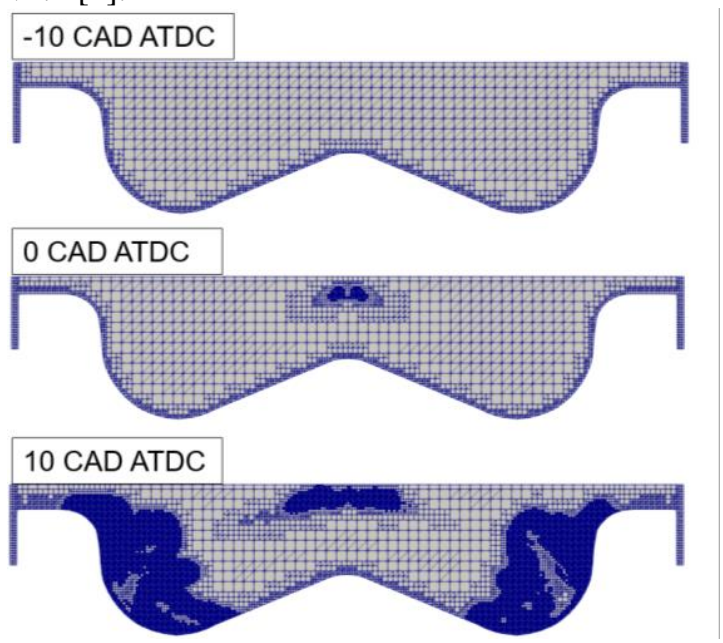

FIGURE 2: TWO-DIMENSIONAL SLICE SHOWING THE MESH AT -10, 0 AND 10 CAD ATDC FOR A CASE USING THE CENTRAL INJECTOR ONLY AT SWIRL RATIO 1 


\subsection{Test Conditions}

All simulations in this study were performed on the Volvo D13 heavy-duty truck engine geometry. TABLE 1 contains the standard engine setup used for all cases. The engine speed was kept constant at $1200 \mathrm{rpm}$ for all cases. It is worth noting that the compression ratio is lower than the typical Volvo D13 engine value. The 11.5:1 compression ratio was found optimal for the DCEE concept [14], which motivated the choice in current simulations.

TABLE 1: GENERAL ENGINE SPECIFICATIONS AND SETUP

\begin{tabular}{|l|l|}
\hline Engine parameters & \\
\hline Cylinder Volume & 2.331 \\
\hline Stroke & $158 \mathrm{~mm}$ \\
\hline Bore & $131 \mathrm{~mm}$ \\
\hline Connecting Rod & $267.5 \mathrm{~mm}$ \\
\hline Compression Ratio & $11.5: 1$ \\
\hline Engine Speed & $1200 \mathrm{rpm}$ \\
\hline Nozzle Hole Diameter & $240 \mu \mathrm{m}$ \\
\hline Injector Umbrella Angle & 145 degrees \\
\hline Intake Pressure & 5 bar \\
\hline Fuel & Diesel \\
\hline
\end{tabular}

Two primary load conditions were evaluated in this study: a medium load and a high load case. TABLE 2 shows the casespecific setup where the high load case included high EGR levels. The EGR was simulated by adding additional $\mathrm{CO}_{2}, \mathrm{~N}_{2}$, and $\mathrm{H}_{2} \mathrm{O}$ to the in-cylinder gas composition at the start of compression.

\section{TABLE 2: CASE SPECIFIC ENGINE SETUP}

\begin{tabular}{|l|l|l|}
\hline & Medium Load & High Load \\
\hline Lambda & 3.2 & 1.4 \\
\hline EGR & $0 \%$ & $36.4 \%$ \\
\hline Intake Temperature & $464 \mathrm{~K}$ & $400 \mathrm{~K}$ \\
\hline Fuel Mass per Cycle & $150 \mathrm{mg}$ & $275.6 \mathrm{mg}$ \\
\hline FuelMEP & $30 \mathrm{bar}$ & $55 \mathrm{bar}$ \\
\hline Injection Pressure & $1500 \mathrm{bar}$ & $2200 \mathrm{bar}$ \\
\hline Start of Injection (SOI) & $-1 \mathrm{CAD}$ ATDC & $-2.7 \mathrm{CAD}$ ATDC \\
\hline Injection Duration & $7.2 \mathrm{CAD}$ & $12.8 \mathrm{CAD}$ \\
\hline
\end{tabular}

TABLE 3 highlights the evaluated cases. The medium load case contained five different swirl ratios at $0,1,2,3$, and 5 chosen as typical swirl ratios for compression-ignition engines with one outlier. The swirl ratios were labeled NS (no swirl), S1 (swirl ratio 1), S2 (swirl ratio 2), S3 (swirl ratio 3), and S5 (swirl ratio 5).

Every swirl ratio included simulations with one, two, and three injectors. FIGURE 3 displays the injector configurations as follows: the reference case refers to the utilization of the central injector only; the two-injector case refers to the utilization of the two outer injectors only; and the three-injector case refers to the simultaneous use of all three injectors.

All evaluated cases employ a single injection divided between the injectors in the following manner: the reference case injects all fuel from the central injector; the two-injector case divides the fuel equally for each side injector; and the threeinjector case injects half the fuel from the central injector and half the fuel from the two side injectors.

The central injector wields six holes of $240 \mu \mathrm{m}$ each, and the side injectors wield three holes, each of the same diameter. Thus, the central injector flow rate is twice the side injector flow rate. The three-injector cases halve the injection duration to keep a constant flow rate.

The two-injector -and three-injector cases included injection against the swirl direction (see FIGURE 3) for the swirl ratio 2. These cases applied the abbreviation AS2 (injection against the swirl with the ratio 2).

The high load case simulated the swirl ratios 0 and 2 for injection with the swirl direction. The high load cases follow as: reference cases at NS; two-injector cases at NS and S2; and three-injector cases at NS and S2.

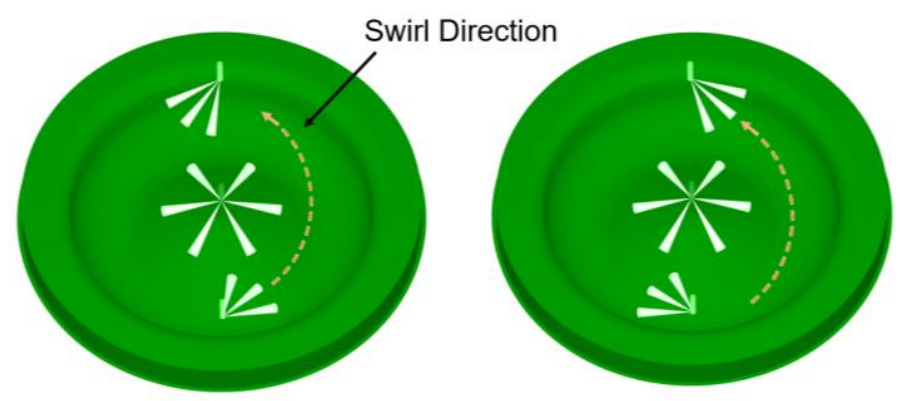

FIGURE 3: INJECTOR AND SPRAY CONFIGURATION INDICATING INJECTION WITH (LEFT) AND AGAINST (RIGHT) THE SWIRL

\section{TABLE 3: EVALUATED CASES}

\begin{tabular}{|c|c|c|c|}
\hline $\begin{array}{c}\text { Swirl } \\
\text { Ratio }\end{array}$ & Load & $\begin{array}{c}\text { No. of } \\
\text { Injectors }\end{array}$ & $\begin{array}{c}\text { Injection Direction } \\
\text { Relative to Swirl }\end{array}$ \\
\hline 0 & Middle/High & $1,2,3$ & With \\
\hline 1 & Middle & $1,2,3$ & With \\
\hline 2 & Middle/High & $1,2,3$ & With/Against \\
\hline 3 & Middle & $1,2,3$ & With \\
\hline 5 & Middle & $1,2,3$ & With \\
\hline
\end{tabular}

\subsection{Validation}

Two different cases used a single-injector approach to validate the simulations. These cases were compared with experimental data in terms of in-cylinder pressure and heat release.

Both cases followed a similar validation procedure. At first, the compression ratio was adjusted to match the experimental motored pressure traces. EGR and residual gases were then 
added to ensure the accuracy of the pressure trace before combustion for the fired cases. Injection timings and injection durations were adjusted to account for the injector's hydraulic delay.

FIGURE 4 shows the in-cylinder pressure and rate of heat release (RoHR) for the medium load experimental and simulation cases. The pressure traces are well-matched, although slightly faster initial combustion is observed for the simulation case due to the filtered experimental pressure trace. FIGURE 5 shows that the pressure and RoHR are well matched for the high load case, although a slightly higher peak of RoHR can be observed from the simulation results.

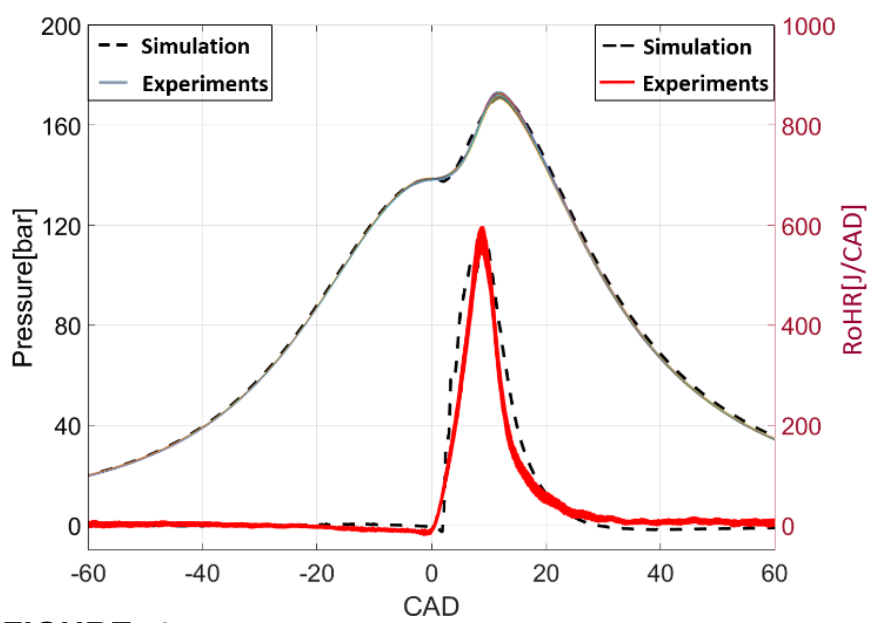

FIGURE 4: EXPERIMENTAL AND SIMULATION PRESSURE [BAR] AND ROHR [J/CAD] FOR THE MEDIUM LOAD VALIDATION CASE

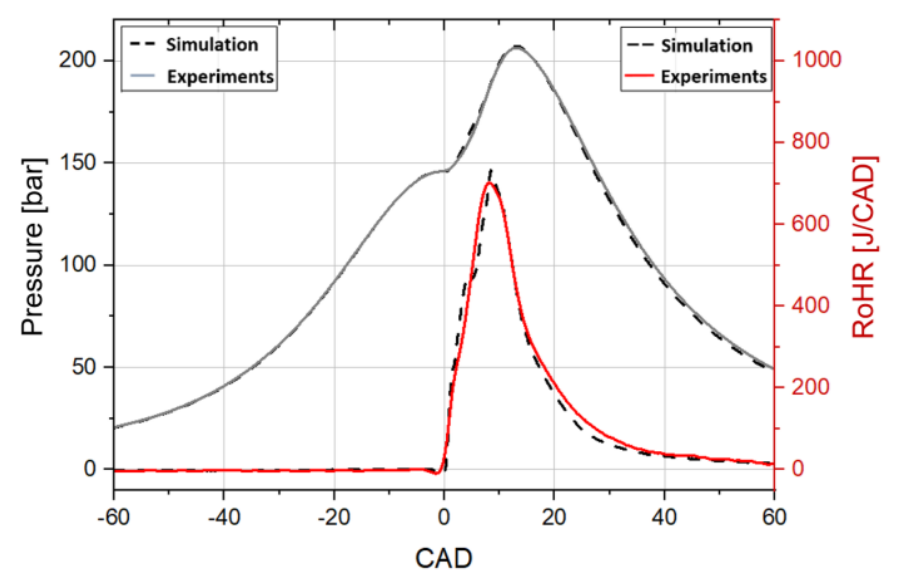

FIGURE 5: EXPERIMENTAL AND SIMULATION PRESSURE [BAR] AND ROHR [J/CAD] FOR THE HIGH LOAD CASE [22]

\section{RESULTS AND DISCUSSION}

\subsection{Efficiency and Performance for Different Swirl Ratios at Medium Load}

FIGURE 6 manifests how an increased swirl reduces the gross indicated efficiency at 30 bar FuelMEP. The multiple injectors limit this trend and flatten the curve, especially at swirl ratios above 2. Movement from no swirl to swirl ratio 5 reduces the indicated efficiency by $2.5 \%$-points, for the reference cases. The same efficiency reduction stays at 2.1 -and $1.7 \%$-points, with two and three injectors, respectively. The use of two injectors increases the indicated efficiency at all swirl ratios except 0 . The swirl ratio 3 realizes the most significant efficiency increase (compared to the reference case) at $0.5 \%$-points. The use of three injectors increases the indicated efficiency at all swirl ratios. The swirl ratio 5 realizes the most significant efficiency increase at $0.9 \%$-points. In conclusion, multiple injectors diminish the efficiency loss at elevated swirl ratios.

FIGURE 7 illustrates the total heat losses during compression and expansion as a function of the swirl ratio. Changing trends are concluded for reference, two-injector -and three-injector cases: the reference -and two-injector cases experience a similar pattern with increased heat losses at an elevated swirl; the three-injector cases experience the same trend, but with a gentler slope. The heat losses change $4.7 \%$ points for the three-injector case from no swirl to swirl ratio 5 . In comparison, the heat losses vary 6.8 -and $7.0 \%$-points for the reference and two-injector cases, respectively. The two-injector cases realize the lowest heat losses at all swirl ratios. In contrast, the three-injector cases achieve lower heat losses, compared to the reference cases, at swirl ratios above 2 .

The DCEE (as described in the introduction) utilizes the exhaust energy to generate work in the expander unit. Since the DCEE expander efficiency depends on load and configuration, FIGURE 8 demonstrates two different example efficiencies. These efficiencies define the potential exhaust energy work output. Reduced heat losses become increasingly important at 75 $\%$ expander efficiency (compared to 50 \%). The DCEE output work (as a fraction of the fuel energy) decreases, from no swirl to the swirl ratio 5 , by $4.7 \%$-points for the reference cases; 4.5 $\%$-points for the two-injector cases; and $3.2 \%$-points for the three-injector cases, with the $50 \%$ expander efficiency. At the $75 \%$ expander efficiency, the same numbers become $5.7 \%$ points for the reference cases; $5.7 \%$-points for the two-injector cases; and $4 \%$-points for the three-injector cases. Finally, the two-injector configurations achieve the highest DCEE work output at all swirl ratios but 5. Thus, the two-injector configuration suits a WHR-capable engine at this load condition.

Injection against the swirl direction reduced the heat losses, as an effect of the effective swirl reduction during the expansion, for both the two-injector -and three-injector configurations (see FIGURE 9). Compared to injection with the swirl, injection against the swirl provided: reduced heat losses by $2.3 \%$-points and increased indicated efficiency by $0.4 \%$-points for the twoinjector configuration; reduced heat losses by $1.9 \%$-points and increased indicated efficiency by $0.6 \%$-points for the threeinjector configuration at the swirl ratio 2 . Thus, injection against the swirl is useful to diminish the experienced high heat losses at elevated swirl ratios. 


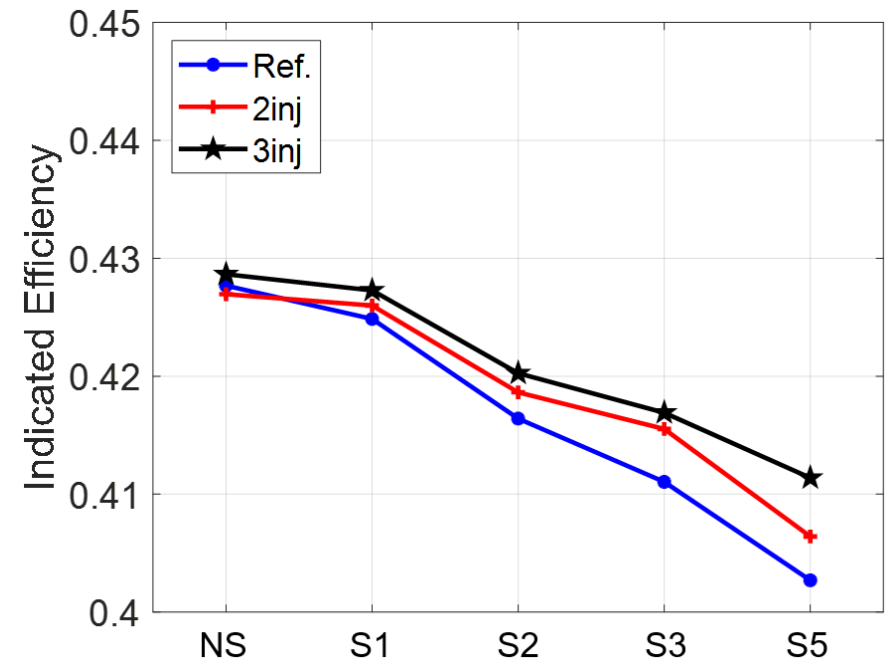

FIGURE 6: GROSS INDICATED EFFICIENCY AS FUNCTION OF SWIRL RATIO FOR THE THREE DIFFERENT INJECTOR CONFIGURATIONS

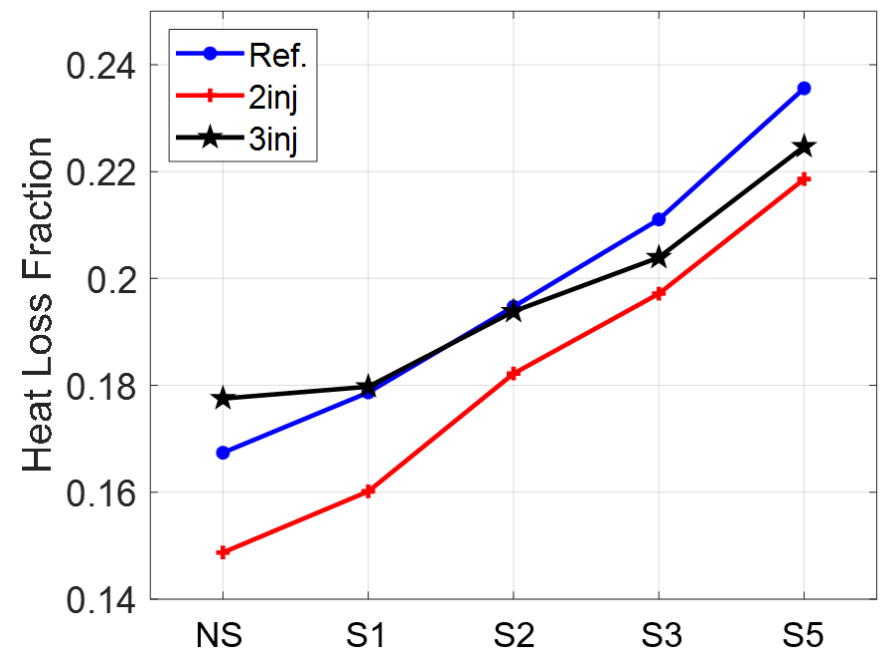

FIGURE 7: TOTAL HEAT LOSSES, DURING THE COMPRESSION AND EXPANSION STROKES, AS FRACTION OF SUPPLIED FUEL ENERGY

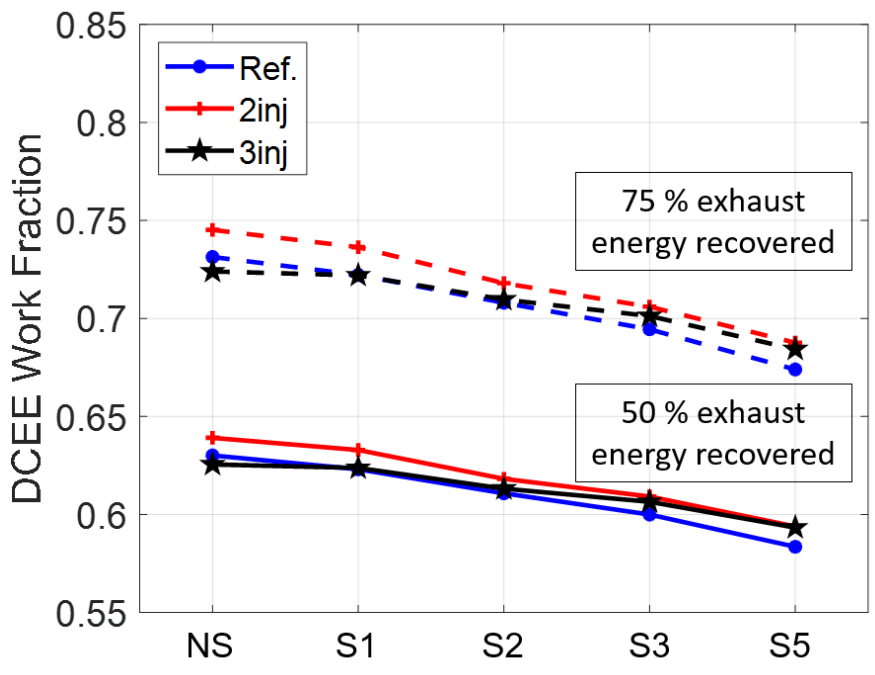

FIGURE 8: OUTPUT WORK AS FRACTION OF SUPPLIED FUEL ENERGY USING THE DCEE FOR EXHAUST ENERGY-TO-WORK CONVERSION AT TWO DIFFERENT EFFICIENCIES

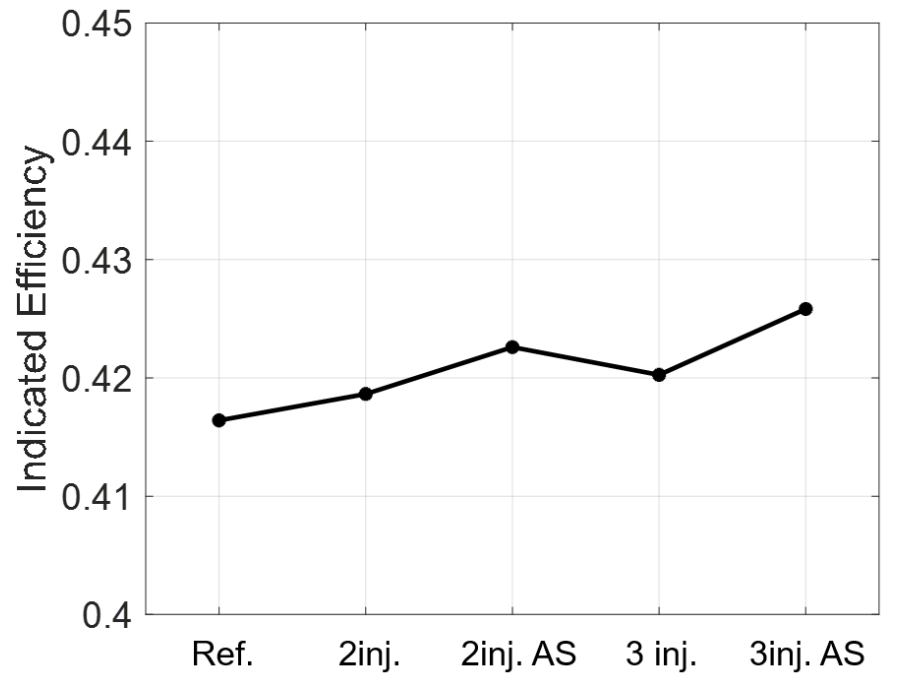

FIGURE 9: GROSS INDICATED EFFICIENCY AS FUNCTION OF INJECTOR CONFIGURATION FOR THE SWIRL RATIO 2

FIGURE 11 to 13 illustrate the pressure and RoHR for three different swirl ratios to provide explanations for the earlier discussed performance. The three-injector cases experience higher initial RoHR as an effect of the increased flow rate. This effect provides the higher indicated efficiency, as concluded in the performance studies above. Here, the high initial RoHR provides high in-cylinder temperatures. Higher temperatures are typically associated with higher convective heat losses, which was not the situation for several three-injector cases. 


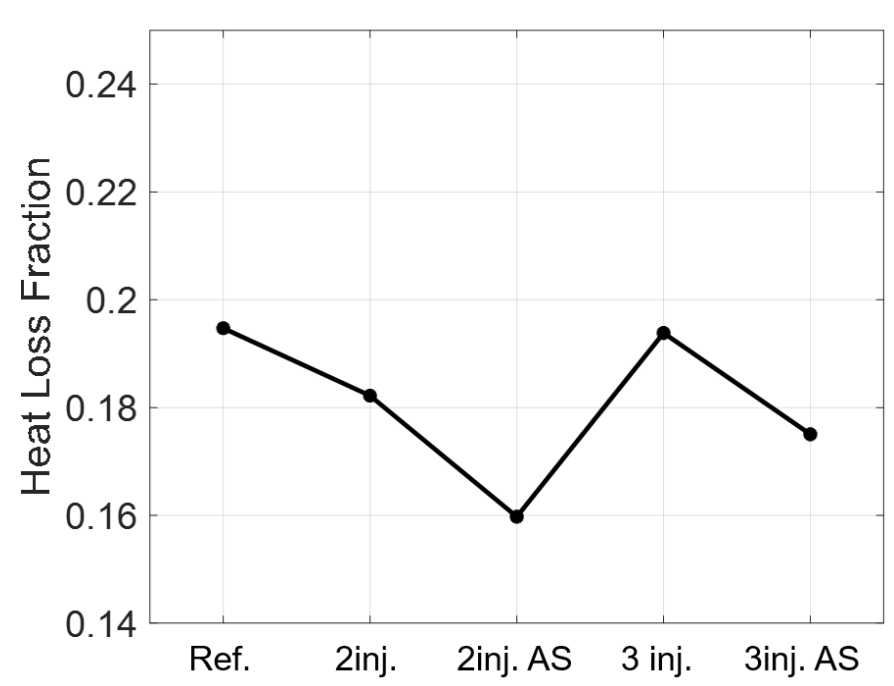

FIGURE 10: TOTAL HEAT LOSSES, DURING THE COMPRESSION AND EXPANSION STROKES, AS FRACTION OF SUPPLIED FUEL ENERGY

The RoHR peak rises with an increased swirl ratio. This rise gives way for higher pressures but also higher heat losses. FIGURE 12 illustrates how injection with the swirl increases the RoHR peak, while injection against the swirl deteriorates early combustion. The slightly slower combustion is one of the explanations (as an addition to the reduced effective swirl discussed before) for the lower heat losses experienced for the two-injector AS2 case. A similar trend is observed where the three-injector AS2 case reduces the early RoHR compared to the three-injector S2 case.

The three-injector configuration improves the chamber utilization compared to the reference -and two-injector cases (see FIGURE 14). However, the three-injector configuration renders more spray-to-spray interaction, especially at high swirl, possibly leading to fuel-rich combustion. The higher flow-rate of the three-injector configuration results in higher local temperatures at 5 CAD ATDC.

As discussed, higher temperatures give higher heat losses. However, the hot zones are centralized to a larger extent for the three-injector cases (compared to the reference cases), leading to smaller temperature gradients at the wall. In comparison, the reference cases experience a large portion of hot zones by the walls. Only minor changes between the different swirl ratios could be observed. Thus, this load condition concluded no significant benefits of increased swirl.

Injection against the swirl renders a shorter jet penetration for the two-injector configuration (see FIGURE 15), leading to hot zones reaching the walls to a smaller extent. Thus, a lower wall temperature gradient and lower convective heat losses follow. The three-injector case experiences small differences only when injecting against the swirl.

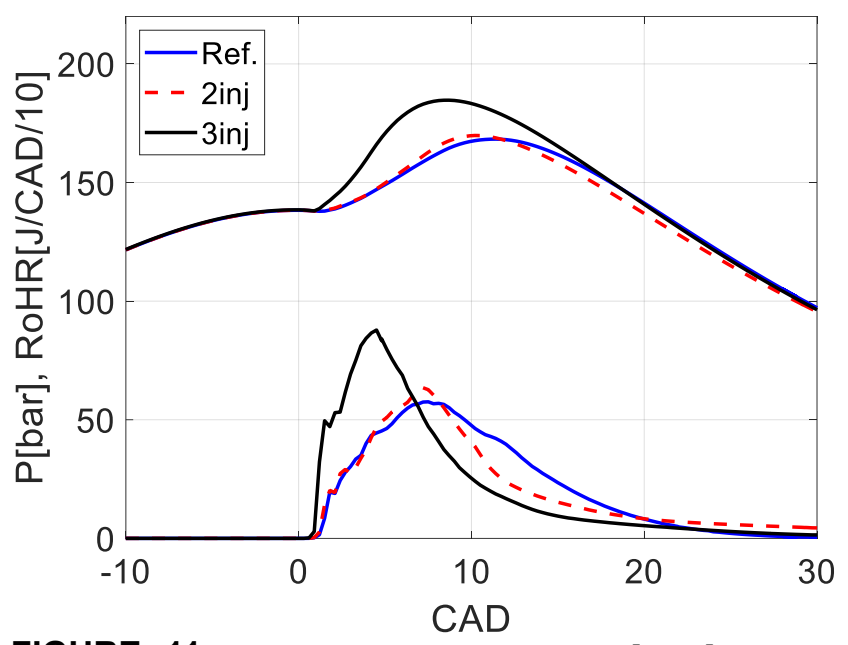

FIGURE 11: IN-CYLINDER PRESSURE [BAR] AND RoHR [J/CAD/10] FOR THE NO SWIRL CASES

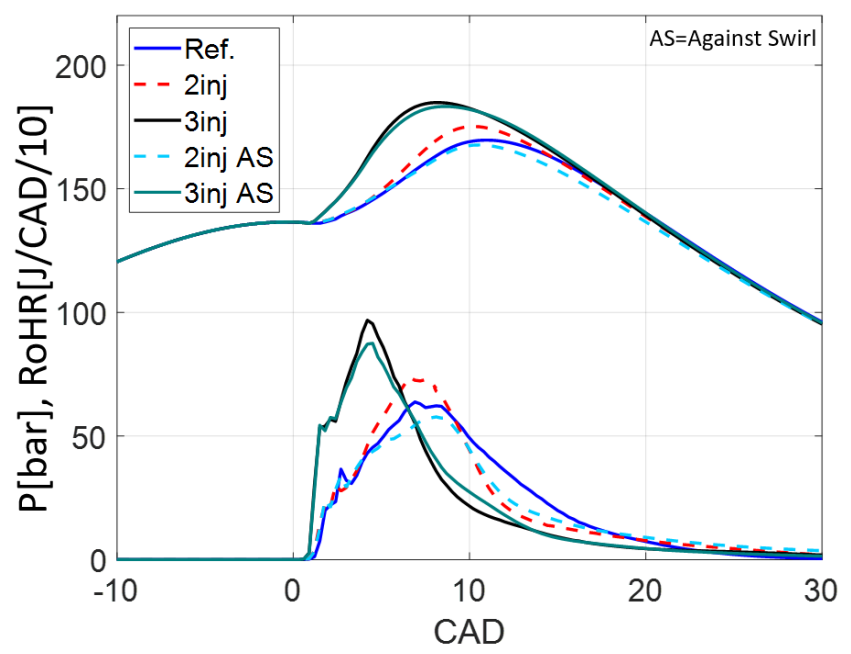

FIGURE 12: IN-CYLINDER PRESSURE [BAR] AND RoHR $[\mathrm{J} / \mathrm{CAD} / 10]$ FOR THE SWIRL RATIO 2 CASES

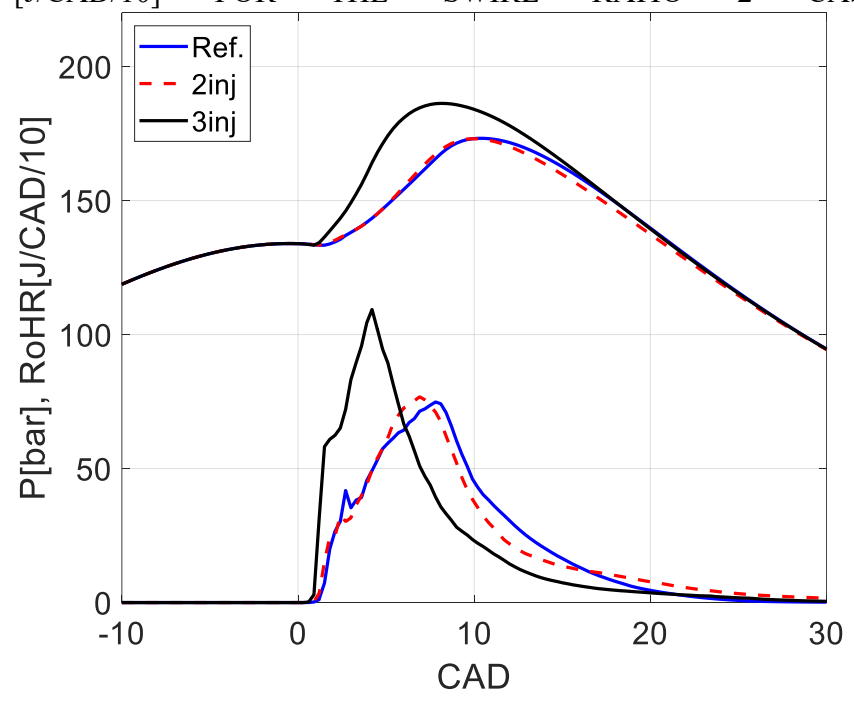

FIGURE 13: IN-CYLINDER PRESSURE [BAR] AND RoHR [J/CAD/10] FOR THE SWIRL RATIO 5 CASES 


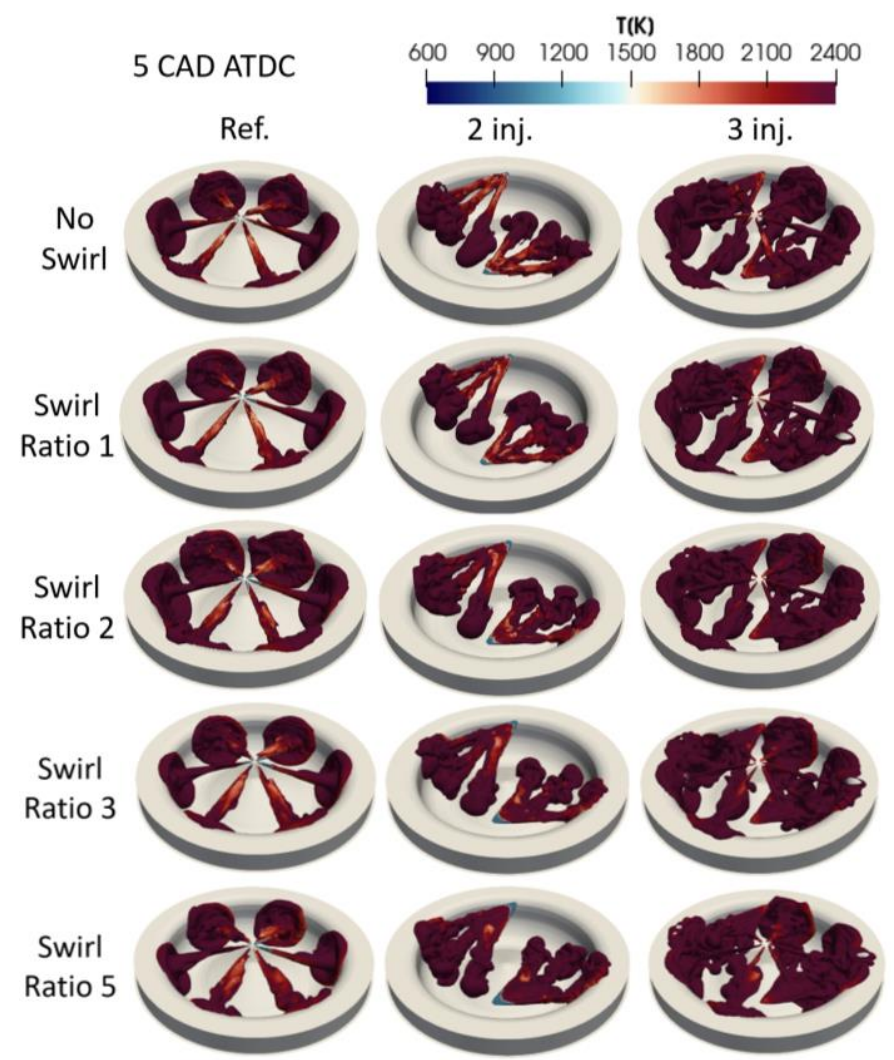

FIGURE 14: CONTOURS, FOR A LOCAL EQUIVALENCE RATIO ABOVE ONE, COLORED BY LOCAL TEMPERATURE [K] FOR ALL CASES INJECTING WITH THE SWIRL

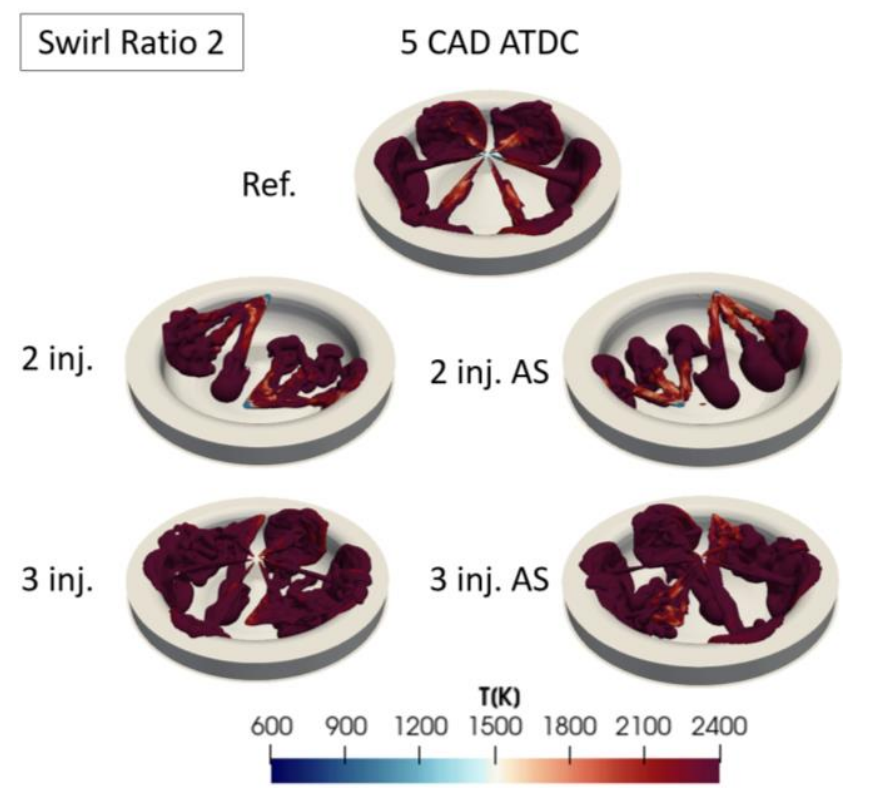

FIGURE 15: CONTOURS, FOR A LOCAL EQUIVALENCE RATIO ABOVE ONE, FOR THE SWIRL RATIO 2 CASES INDICATING DIFFERENCES WHEN INJECTING AGAINST THE SWIRL DIRECTION
As a summary of the performance section, the threeinjector cases provided high indicated efficiencies with lower heat losses for elevated swirl ratios. The two-injector cases delivered the most moderate heat losses and thus suited WHR engines. Injection against the swirl reduced the heat losses and increased the indicated efficiency by reducing the effective swirl during the expansion stroke. A high swirl ratio decreased the efficiency, from higher heat losses, unless specific multiple injector configurations were utilized. Finally, multiple injectors lead to higher engine production costs. Thus, the performance benefits (emissions and fuel consumption) should outweigh the increased costs for a future production engine.

\subsection{Multiple Injector Load Dependency}

FIGURE 16 shows the mean effective pressures for several high load cases (55 bar FuelMEP). With the higher fuel amount, the combustion losses increase with a maximum of $0.9 \%$ for the two-injector NS case. A higher swirl mitigates the combustion losses falling to $0.5 \%$ for the two-injector S2 case. The use of three injectors significantly lowers combustion losses (see FIGURE 16).

The reference NS case renders the highest indicated efficiency at $43 \%$, followed by the three-injector NS case at 42.9 $\%$. The indicated efficiency is reduced to the lowest level of 39.1 $\%$ for the two-injector NS case by the higher combustion losses and deteriorated thermodynamic cycle.

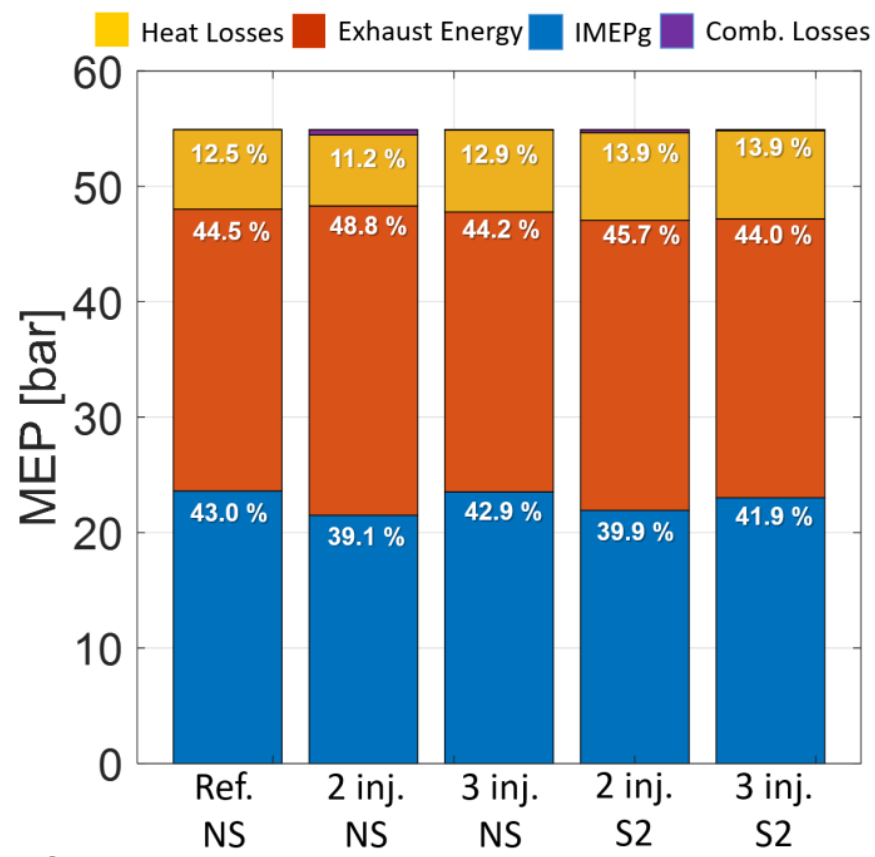

FIGURE 16: MEAN EFFECTIVE PRESSURES [BAR], FOR THE HGH LOAD, IN TERMS OF HEAT LOSSES, EXHAUST ENERGY, GROSS INDICATED WORK AND COMBUSTION LOSSES. NS=NO SWIRL; S2=SWIRL RATIO 2 
The heat losses decrease by $1.3 \%$-points for the twoinjector NS case as an effect of the low combustion efficiency. Otherwise, the NS reference case experiences the lowest heat losses at $12.5 \%$. The NS two-injector case renders the highest combined indicated efficiency and exhaust energy levels at 87.9 $\%$. It might thus be more suitable when utilizing a WHR system at this load condition.

FIGURE 17 confirms the lower combustion efficiency for the two-injector cases. The two-injector RoHR follows the reference case RoHR until 5 CAD ATDC. At 5 CAD ATDC, the combustion decays. Again, the high flow rate of the threeinjector cases provides a fast early RoHR. This results in a rapid pressure rise giving a longer effective expansion stroke. However, the higher combustion losses prevent a higher work output than the reference case.

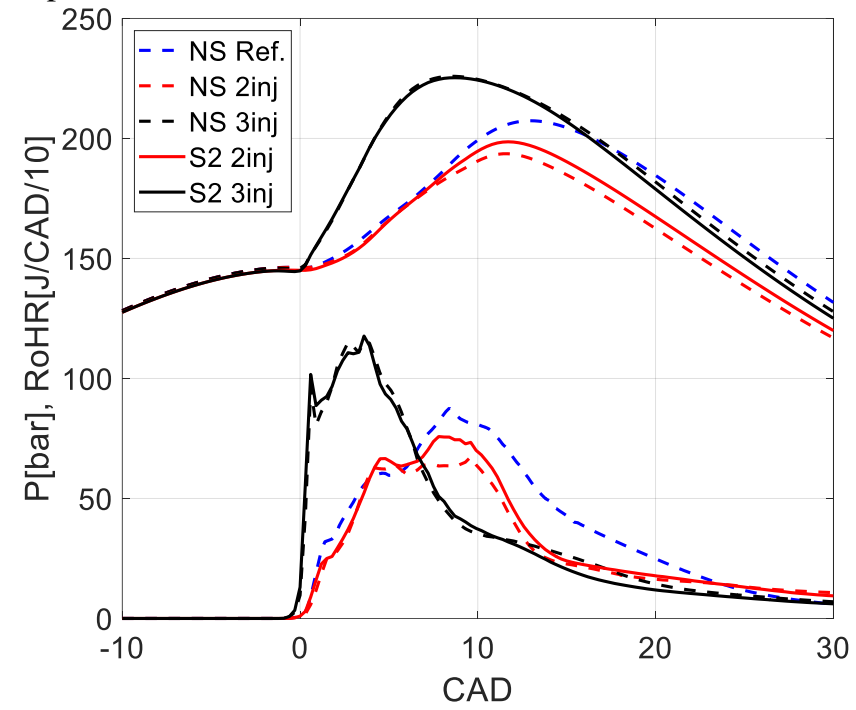

FIGURE 17: IN-CYLINDER PRESSURE [BAR] AND RoHR [J/CAD/10] FOR THE HIGH LOAD CASES

FIGURE 18 illustrates the combustion issues addressed above. Large amounts of spray to spray interference are observed for the 55 bar FuelMEP case, thus explaining the combustion decay at 5 CAD ATDC. The spray interference at the edge of the bowl cannot be observed for the 30 bar FuelMEP case. This is in line with the RoHR where no substantial combustion decay is noted for the two-injector cases at a FuelMEP of 30 bar.
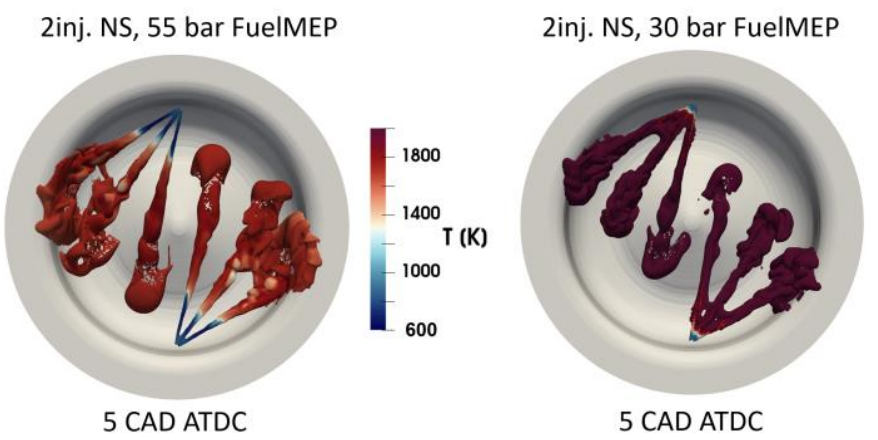

FIGURE 18: CELL CONTOURS WITH AN EQUIVALENCE RATIO ABOVE 2 AND TEMPERATURE COLORING [K] FOR THE TWO DIFFERENT LOAD CASES UTILIZING TWO INJECTORS
In summary, the proposed multiple injector configurations are not as well suited for high load conditions. The injections from the outer injectors suffer from poor combustion efficiency. Thus, the work output levels are also low. Utilizing all three injectors mitigate the combustion problems for the outer injector cases, but a slightly lower work output persists. Finally, future studies are needed on multiple injector system optimizations at high load conditions.

\section{CONCLUSIONS AND DISCUSSION}

This study presents CFD simulations investigating how multiple injectors decrease the high heat losses resulting from elevated swirl in a heavy-duty CI engine. Five different swirl ratios and two load conditions were evaluated with one, two, and three injectors. Higher swirl ratios provided small heat release differences and reduced the indicated efficiency due to high heat losses.

Two-injector utilization provided some benefits at middle load as follows: reduced heat losses for all cases, especially when injecting against the swirl; increased indicated efficiency at several swirl ratios; and high exhaust energy, useful for waste heat recovery systems. Although the two-injector cases increased the efficiency, the no-swirl levels could not be reached at any other swirl ratios. At high load, the two-injector cases provided low heat losses, but low indicated efficiencies resulting from slow heat release.

The three-injector configuration, at middle load, resulted in: reaching the highest efficiencies for all swirl ratios, as a direct effect of the higher flow rate; delivering low heat losses, compared to the reference cases, at swirl ratios above 2; diminishing the exhaust energy compared to the two-injector cases. The three-injector configuration could not recover the lowered indicated efficiency for swirl ratios above 1. At high load, the three-injector configuration improved the drawbacks of the two-injector case and reached similar efficiencies as the reference case.

A summary of the main conclusions is listed below:

1. Utilization of three injectors increased the indicated efficiency for all swirl ratios at medium load up to $0.8 \%$ points.

2. Two-injector configurations reduced the heat losses up to $3.6 \%$-points compared to single-injector cases.

3. Injection against the swirl direction was proved beneficial for multiple injectors.

4. Two-injector setups, combined with a WHR system, gave an efficiency increase of up to $2.2 \%$-points.

5. Three-injector concepts showed potential at high load conditions, but further system optimization is needed. 


\section{ACKNOWLEDGEMENTS}

This work was sponsored by King Abdullah University of Science and Technology (KAUST). The simulations in this work were performed with the computing resources at the KAUST Supercomputing Laboratories. The authors would like to thank Dr. Georgios Markomanolis at KAUST Supercomputing Laboratory for helpful guidance in post-processing, and $\mathrm{Mr}$. Nhut Lam at Lund University for providing the experimental data for model validation.

\section{REFERENCES}

[1] European Commission,

"Reducing CO2 emissions from heavy-duty vehicles",

https://ec.europa.eu/clima/policies/transport/vehic les/heavy_en (accessed 2018-05-10)

[2] European Environment Agency, "Carbon dioxide emissions from Europe's heavy-duty vehicles" https://www.eea.europa.eu/themes/transport/heav $\mathrm{y}$-duty-vehicles/carbon-dioxide-emissions-europe (accessed 2018-09-23)

[3] Delgado, O., Lutsey, N., 2014. The U.S. Supertruck Program Expediting the Development of Advanced Heavy-Duty Vehicle Efficiency Technologies. ICCT

[4] Heywood, John B. "Internal combustion engine fundamentals" McGraw-Hill Book Company 1988

[5] Kook, S., Bae, C., Miles, P., Choi, D. et al., "The Effect of Swirl Ratio and Fuel Injection Parameters on CO Emission and Fuel Conversion Efficiency for High-Dilution, LowTemperature Combustion in an Automotive Diesel Engine," SAE Technical Paper 2006-010197, 2006, https://doi.org/10.4271/2006-010197.

[6] Olmeda, P., Martin, J., Garcia, A., Villalta, D. et al., "A Combination of Swirl Ratio and Injection Strategy to Increase Engine Efficiency," SAE Int. J. Engines 10(3):2017, doi:10.4271/2017-010722.

[7] Fridriksson, H., Tuner, M., Andersson, O., Sunden, B. et al., "Effect of Piston Bowl Shape and Swirl Ratio on Engine Heat Transfer in a Light-Duty Diesel Engine," SAE Technical Paper 2014-01-1141, 2014, https://doi.org/10.4271/2014-01-1141.

[8] Okamoto, T. and Uchida, N., 2016. New concept for overcoming the trade-off between thermal efficiency, each loss and exhaust emissions in a heavy duty diesel engine. SAE International Journal of Engines, 9(2), pp.859-867.

[9] Enya, K., Watanabe, H. and Uchida, N., 2018. Investigation into the Optimized Heat Release Rate and Corresponding Variation of In-
Cylinder Specific Heat Ratio for the Improvement in Thermal Efficiency by Utilizing Two-Zone Combustion Model Analysis (No. 2018-01-1796). SAE Technical Paper.

[10] Nyrenstedt, G., Watanabe, K., Enya, K., Shi, H., Uchida, N. and Johansson, B., 2019. Thermal Efficiency Comparison of Different Injector Constellations in a CI Engine (No. 2019-240172). SAE Technical Paper.

[11] Nyrenstedt, G., Alturkestani, T.L.M., Im, H.G. and Johansson, B., 2019. CFD Study of Heat Transfer Reduction Using Multiple Injectors in a DCEE Concept.

[12] Nyrenstedt, G., Im, H., Andersson, A. and Johansson, B., 2019. Novel Geometry Reaching High Efficiency for Multiple Injector Concepts (No. 2019-01-0246). SAE Technical Paper.

[13] Lam, N., Andersson, A. and Tunestal, P., 2018. Double Compression Expansion Engine Concepts: Efficiency Analysis over a Load Range (No. 2018-01-0886). SAE Technical Paper.

[14] Lam, N., Tuner, M., Tunestal, P., Andersson, A., Lundgren, S. and Johansson, B., 2015. Double compression expansion engine concepts: a path to high efficiency. SAE International Journal of Engines, 8(4), pp.1562-1578.

[15] Reitz, R., and Diwakar, R., "Structure of HighPressure Fuel Sprays," SAE Technical Paper 870598, 1987, doi:10.4271/870598.

[16] Amsden, A. A., O'rourke, P. J., and Butler, T. D., KIVA-II: A computer program for chemically reactive flows with sprays (No. LA-11560-MS). Los Alamos National Lab., NM (USA), 1989.

[17] Torregrosa, A., P. Olmeda, By Degraeuwe, and M. Reyes. "A concise wall temperature model for DI Diesel engines." Applied Thermal Engineering 26, no. 11-12 (2006): 1320-1327.

[18] Amsden, A. A., "KIVA-3V: A Block Structured KIVA Program for Engines with Vertical or Canted Valves," Los Alamos National Laboratory Report No. LA-13313-MS, 1997.

[19]Zeuch, Thomas, Gladys Moréac, Syed Sayeed Ahmed, and Fabian Mauss. "A comprehensive skeletal mechanism for the oxidation of $n$-heptane generated by chemistry-guided reduction." Combustion and Flame 155, no. 4 (2008): 651-674.

[20] Babajimopoulos, A., Assanis, D. N., Flowers, D. L., Aceves, S. M., et. al., "A fully coupled computational fluid dynamics and multi-zone model with detailed chemical kinetics for the simulation of premixed charge compression ignition engines," International journal of engine research, 6:497-512, 2005, doi:10.1243/146808705X30503. 
[21] Richards, K. J., Senecal, P. K., and Pomraning, E., "CONVERGE (Version 1.4.1) Manual,"

Convergent Science, Inc., Middleton, WI, 2012.
[22] Babayev, R., Nyrenstedt, G., Johansson, B., et. al. 2019. Computational Study of a Multiple Fuel Injector Concept under High-Load and High-EGR Conditions. SAE Technical Paper 2020-01-2034. 